\title{
Green Nail Syndrome caused by Citrobacter braakii
}

\author{
Francesk Mulita ${ }^{1}$, Nikolaos Parchas $^{1}$, Levan Tchabashvili ${ }^{1}$, Elias Liolis ${ }^{1}$, Fotis Iliopoulos ${ }^{1}$, \\ and Kerasia-Maria Plachouri ${ }^{1}$ \\ ${ }^{1}$ University General Hospital of Patras Holy Mary the Help
}

March 19, 2021

\begin{abstract}
A 34-year-old woman presented due to progressive painful swelling around the nail of the right index finger. Onychectomy and drainage of the abscess of the affected finger were performed as the inflammation was progressive despite the previous antibiotic therapy. The microbiological culture revealed a ciprofloxacin-susceptible Citrobacter braakii.
\end{abstract}

CLINICAL IMAGE

\section{Green Nail Syndrome caused by Citrobacter braakii}

\section{Key Clinical Message}

Green nail syndrome (GNS) is a rare condition, usually caused by Pseudomonas aeruginosa. A case of GNS caused by $C$. braakii has not been reported in the literature so far.

\section{Case Description}

A 34-year-old woman presented in our emergency department due to progressive painful swelling around the nail of the right index finger, that had first appeared 30 days prior to the referral. The symptoms occurred immediately after a finger injury. Approximately 2 weeks after the trauma, the patient noticed a greenish discoloration of the nail plate of the affected finger. An empirical antibiotic therapy with amoxicillin/clavulanic acid had been unsuccessful.

On examination, the patient's vital signs were unremarkable. A distal onycholysis and a greenish discoloration of the entire nail plate of the right index finger, as well as a purulent proximal nail fold were seen (Figure 1).

Onychectomy and drainage of the abscess of the affected finger were performed as the inflammation was progressive despite the previous antibiotic therapy. The microbiological culture revealed a ciprofloxacin-susceptible Citrobacter braakii (C. braakii ) infection and a postoperative antibiotic therapy with ciprofloxacin was therefore prescribed. In the five-day follow up, the patient was symptom-free and the inflammatory markers (WBC, CRP) were within the normal range.

Green nail syndrome (GNS) is a rare condition, usually caused by Pseudomonas aeruginosa [1]. However, other pathogens, such as the rare gram-negative bacillus C. braakii can also be involved in the pathogenesis of this condition [2].

Keywords: Green nail syndrome, onycholysis, onychectomy,Citrobacter braakii

\section{Patient consent for publication}

A written informed consent was obtained from the patient for publication of this case report. 


\section{Conflict of interest}

There are no conflicts of interest to declare.

Financial support and sponsorship

None

\section{Author contribution}

FM, NP, EL, LT, FI and K-MP: contributed to the clinical data collection and prepared the case report. FM, NP and K-MP: contributed to the design of the case report presentation and performed the final revision of the manuscript.

\section{Data availability}

Data available on request from the authors

\section{References}

Schwartz RA, Reynoso-Vasquez N, Kapila R. Chloronychia: The Goldman-Fox Syndrome - Implications for Patients and Healthcare Workers. Indian J Dermatol . 2020;65(1):1-4.

Oyeka M, Antony S. Citrobacter braakii Bacteremia: Case Report and Review of the Literature. Infect Disord Drug Targets. 2017;17(1):59-63. 


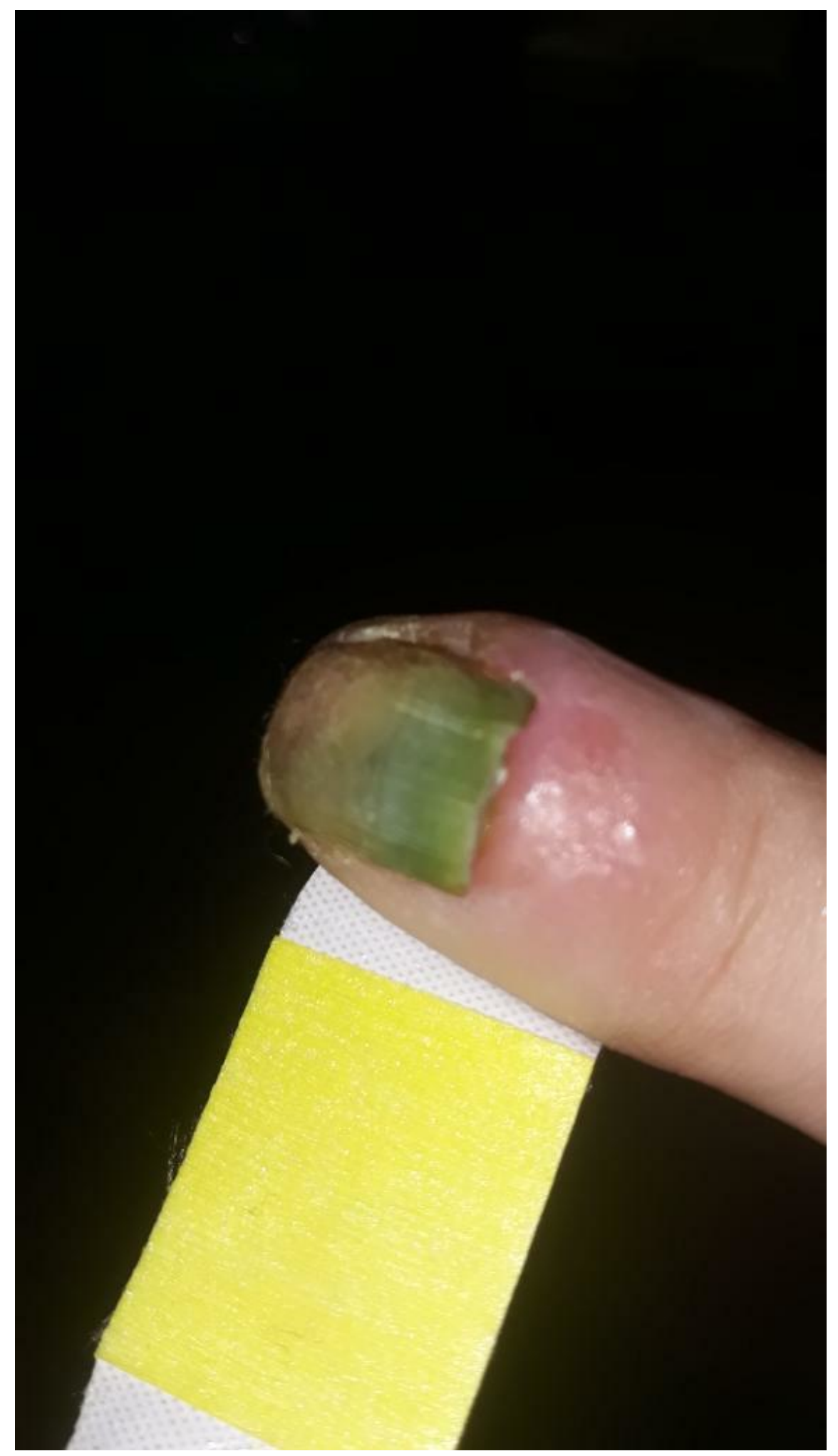

Figure 1 : Green Nail Syndrome in a 34-year young woman: A distal onycholysis and a greenish discol- 
oration of the entire nail plate of the right index finger, as well as a purulent proximal nail fold.

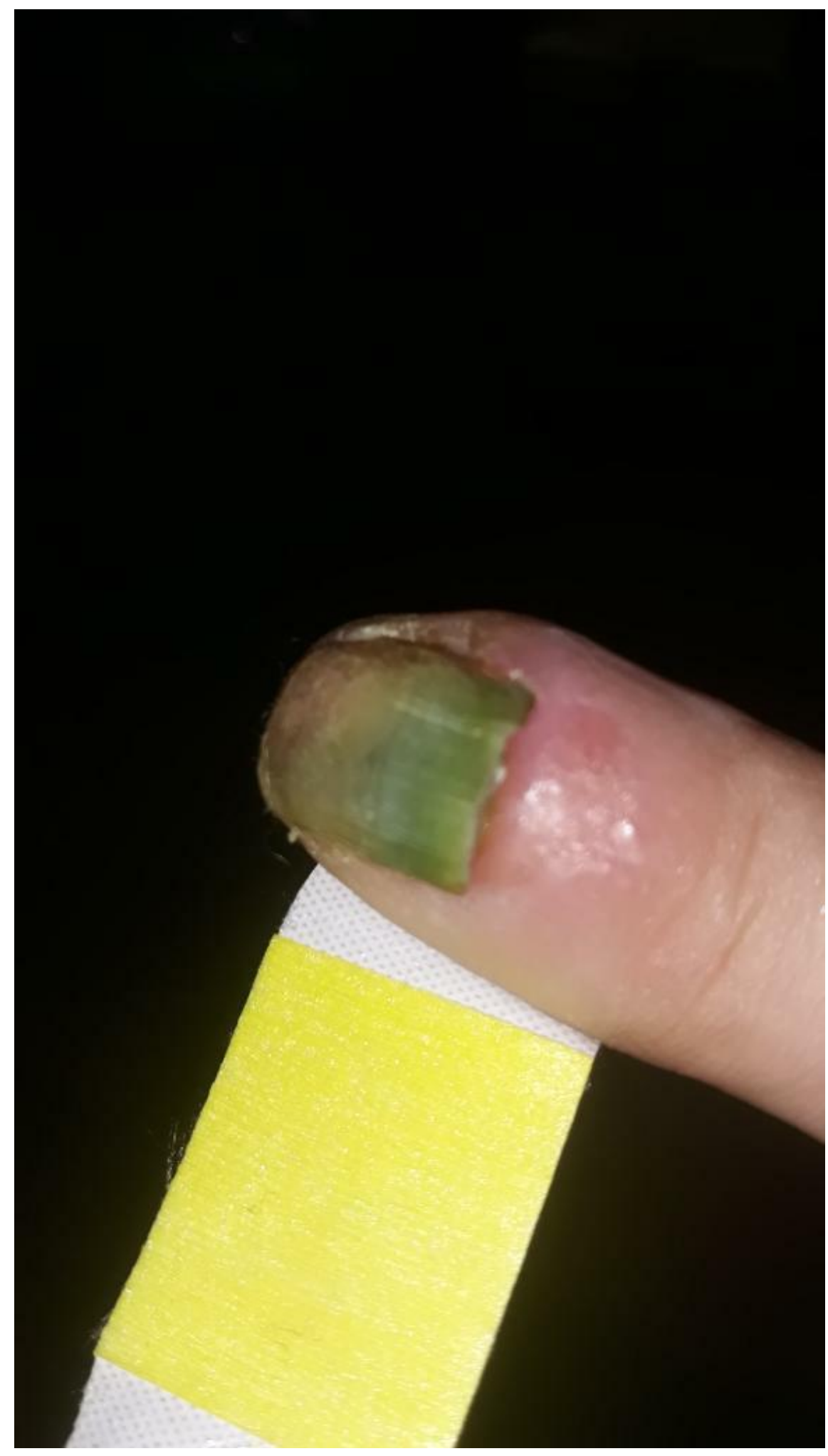

